\title{
Spallation behaviour of a Zr-bulk metallic glass
}

\author{
Z. Ling, X. Huang, L.T. Shen, and L.H. Dai \\ State Key Laboratory of Nonlinear Mechanics (LNM) Institute of Mechanics, Chinese Academy of Sciences, \\ Beijing 100190, PR China
}

\begin{abstract}
Plate impact experiments have been conducted on a Zr-based bulk metal glass (BMG) using a single stage light gas gun. To understand the spallation process of the material, samples were subjected to dynamic tensile loadings of the same amplitude but different durations. Fractographs of spallation surface and fracture features were characterized and the fracture mechanism of different regions of the spallation surface was discussed. Morphology of the spallation surface in the $\mathrm{Zr}$-BMG exhibited a typical equiaxial cellular pattern and porous microstructure. These experiments revealed that, subjected to hydrotensile stresses, the microdamage of the spallation occurred in the $\mathrm{Zr}$-BMG is microvoids; the spallation in the $\mathrm{Zr}$-BMG is resulted from nucleation, growth and coalescence of microvoids; and the time needed for these microvoids nucleation is less than $100 \mathrm{~ns}$ with a stress amplitude of $3.18 \mathrm{GPa}$.
\end{abstract}

\section{Introduction}

Spallation is a typical fracture phenomenon occurred in solids under impulsive loadings. It is recognized as the emergence of distributed microcracks or microvoids, in a narrow region of the material, resulting in catastrophic failure in the material undergoing millions of microdamage (microcracks or microvoids) nucleation, growth and coalescence [1-3]. Essentially, physical characterizing microdamage evolution of the spallation is usually performed experimentally by means of gas-gun driven plate-impact tests, controlling stress amplitude or stress duration to trace the microdamage evolution in laboratories. In the tests, stress durations can simply be made as short as the time required for microdamage nucleation, growth and coalescence till damages are caught up, or being "frozen", at each stage of this damage development [1,4-7].

So far, studies on spallation microdamage evolutions are performed on crystalline metals or metal matrix composites etc, revealing micromechanisms of nucleation, growth and coalescence of the microdamage [1]. The researches have indicated that, microdamage of spallation occurred in tested materials always nucleate at microscopic heterogeneities in the materials, such as pre-existed flaws, second phases, inclusions or grain boundaries etc. Bai et al [4] investigated the initial development of microdamage from spallation occurred in an Al-alloy. The microdamage, microcracks, appeared always at interfaces between the second phase particles and the matrix. The initial development of such microcracks depends on the second phase particles [4]. However, Li studied microdamage evolution of spallation in copper and found out that the microdamage in the tested copper was microvoids [5]. Ling and Shen reported spallation evolution tests on a metal matrix composite and found out that microcracks would nucleate at the interface between reinforced particles and the matrix, while the stress sustains longer, such microcracks start growing [8]. According to what Curran et al. pointed out, in these crystalline materials, the basic faults are crystal dislocations. Therefore, microdamage will nucleate while the dislocations are arrested or pile up at the microscopic heterogeneities under tensile stress or inelastic deformation. As reported in cases of crystalline materials, the scale of nucleated microdamage is consistent with characteristic microstructure scales of the material, i.e. scales of grains or of second phases in alloys, or scales of reinforced phases or of matrix grains in composites $[4,5,8]$. Moreover, the mechanisms of growth and coalescence of such microvoids or microcracks in crystalline materials are intimately related to basic faults, i.e. dislocations [1] in the material.

Bulk metallic glasses (BMGs) have received wide attention because of their excellent mechanical properties and functional applications. Due to the unique amorphous structure and a lack of long-range order in the atomic structure [9], micro-mechanisms of inelastic deformation of BMGs are no longer related to dislocation-based mechanisms that always characterize the plastic deformation of crystalline metals. Due to the unique amorphous structure, BMGs' failure exhibits unusual behaviours, quite different from crystalline materials. These behaviours are being investigated with a considerable interest. A concept of "free volume" [10-12], has currently been applied to understand BMGs' fracture and failure behaviours under quasistatic and dynamic compressive, as well as shear loading [13-17].

Recently, spallation behaviours in BMGs have been reported [18-20] on spallation strength of BMGs, spallation setup occurred in BMGs probed during plate-impact tests and microscopic characteristics for spalled surfaces as well. Zhuang et al [18] examined shock response in $\mathrm{Zr}$ BMG and its composites. By employing VISAR system to obtain the particle velocity history in the samples, resultant spallation and pall strength of the samples were investigated. The spallation occurred in the samples of Vit 1 was regarded as being induced by shear localization, while in those of $\beta$-Vit, the spallation occurred was due to debonding of the $\beta$-phase boundary from the matrix [18]. Turneaure et al [19], applying the VISAR system, studied spallation strength of BMGs at peak stresses below the Hugoniot elastic limit (HEL), revealing an onset stress for fracture in the spalling samples and a change from ductile to brittle tensile behaviour with increasing compressive 
Table 1. Mechanical properties of the Zr-BMG samples.

\begin{tabular}{lcccccc}
\hline Materials & $\rho\left(\mathrm{kg} / \mathrm{m}^{3}\right)$ & $\mathrm{E}(\mathrm{Gpa})$ & $\mathrm{G}(\mathrm{Gpa})$ & $v$ & $\mathrm{c}_{\mathrm{L}}(\mathrm{m} / \mathrm{s})$ & $\mathrm{c}_{\mathrm{t}}(\mathrm{m} / \mathrm{s})$ \\
\hline Zr-BMG & 6125 & 98.6 & 35.3 & 0.36 & 5182 & 2464 \\
\hline
\end{tabular}

stress as well. Yuan et al [20] investigated influences to spallation strength of BMGs under impact loading combined compression-shear and reported an unusual trend of the BMG's spallation strength under shear strain. More recently, Escobedo and Gupta [21] studied fracture mechanisms for spallation in BMG samples (BBA). Based on fracture morphology observations to the spallation surface of the samples, they suggested a mechanism of the arrest and propagation of mode I cracks.

However, the micro-mechanism involved in microdamage evolution, nucleation, growth and coalescence of spallation in BMGs, is still largely unclear. "Not only are the constitutive relations describing microstatistical spallation processes highly nonlinear and therefore resistant to analysis, but there has also been a lack of accurate data for the voids and cracks" [1], as being indicated by Curran et al. in 1987. This statement still rightly holds to current studies of the microdamage evolution of spallation occurred in BMG materials. As BMGs do not exhibit a longrange translational symmetry, a question remains whether it still holds true that the spallation in such materials is resulted from nucleation, propagation and coalescence of microdamage evolution, like those occurred in crystalline materials. If this is true, what is the microdamage of the spallation in the BMGs, microvoids or microcracks?

To better understand BMGs' spallation behaviour and micromechanism, we in this paper report spallation occurred in a Zr-BMG material under plate-impact loading. To reveal microdamage evolution process of spallation in the material, a specially designed loading apparatus, with the same stress amplitude under various stress durations, was adopted to carry out dynamic plate-impact tests.

All the recovered samples were investigated by scanning electron microscope (SEM) and an optical microscope $(\mathrm{OM})$ to reveal spallation surface morphology and the fracture features. Based on the morphology of the spallation surface of the samples and deformation mechanisms related to the intrinsic faults of free volume in the tested $\mathrm{Zr}-\mathrm{BMG}$, a possible nucleation mechanism of spallation is proposed and discussed.

\section{Experimental procedures}

\subsection{Materials and spallation experiments}

The material applied in current experiments was $\mathrm{Z}_{\mathrm{r} 41.2} \mathrm{Ti}_{13.8} \mathrm{Cu}_{12.5} \mathrm{Ni}_{10} \mathrm{Be}_{22.5}$ [Vit.1] and BMG samples were identified to be in full amorphous state by conventional $\mathrm{x}$-ray diffraction before spallation experiments. Table 1 presents mechanical properties of $\mathrm{Zr}$-BMG, where $\rho$ density; E elastic modulus and G shear modulus; $v$ Poisson's ratio; $c_{\mathrm{L}}$ longitudinal wave speed and $\mathrm{c}_{t}$ shear wave speed.

Spallation microdamage evolution tests were performed with a 101-single-stage light gas gun. To follow the evolution of microdamage occurred in the tested material
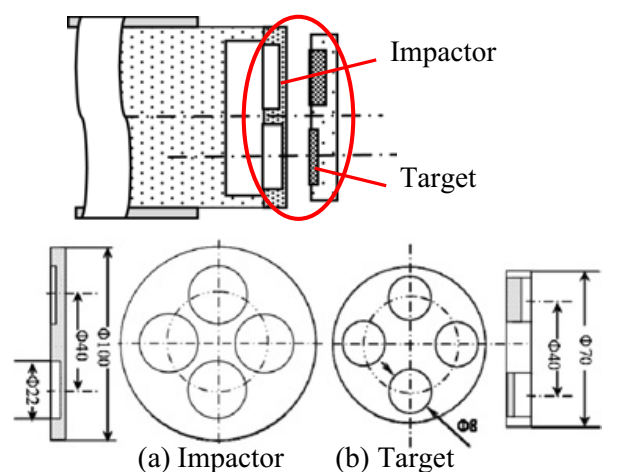

Fig. 1. A schematic of the special designed planner impact apparatus.

Table 2. Spall tests data $\left(\mathrm{V}=200 \mathrm{~m} / \mathrm{s} ; \sigma_{\text {tensile }}=3.18 \mathrm{GPa}\right.$.

\begin{tabular}{lcccc}
\hline Sample & ${ }^{\mathrm{a}} \mathrm{h}(\mathrm{mm})$ & ${ }^{\mathrm{b}} \delta(\mathrm{mm})$ & $\Delta \mathrm{t}(\mathrm{ns})$ & $\sigma_{\text {tensile }}$ \\
\hline $1-1$ & 0.585 & 1.04 & 201 & spalled \\
$1-2$ & 0.45 & 0.90 & 174 & cracks \\
$1-3$ & 0.335 & 0.64 & 123 & N.A. \\
$1-4$ & 0.135 & 0.43 & 83 & cracks \\
$2-1$ & 0.585 & 1.03 & 199 & spalled \\
$2-2$ & 0.45 & 0.85 & 164 & no crack \\
$2-3$ & 0.335 & 0.63 & 122 & N.A. \\
$2-4$ & 0.135 & 0.44 & 85 & no crack \\
\hline
\end{tabular}

${ }^{\mathrm{a}} \mathrm{h}=$ flyer thickness; ${ }^{\mathrm{b}} \delta=$ target thickness.

subjected to impulse loading, the multi-pulse approach for spallation evolution was applied [6-8]. In this technique, several samples were impacted by several flyers with different thickness under one shot, so that progressively damaged samples under the same impact speed and different pulse durations were obtained. Figure 1 shows a schematic of the plate-impact apparatus, where figure $1 \mathrm{a}$ and $1 \mathrm{~b}$ are specially designed flyers and targets, respectively, for the multi-pulse approach. As is shown in the figure, in the impactor, four small flyers with different thickness can yield various pulse durations during the impact. Four small targets were at various positions corresponding to where those four flyers were placed, respectively. The projectile rotation during launching in the gun tube was less than $1^{\circ} \sim 2^{\circ}$ which guaranteed the success of simultaneous measurements of four profiles in one shot.

Table 2 lists current tested samples' parameters, where $\mathrm{h}$ is flyers' thickness, $\delta$ targets' thickness, $\Delta \mathrm{t}$ stress duration and $\sigma$ tensile stress amplitude. The BMG samples were machined to disks of $8 \mathrm{~mm}$ in diameter. Thicknesses of the targets and flyers were designed in accordance with 1-D strain state during impact. The stress durations in current spallation tests were designed as a few tenths of a microsecond, 101-2 ns, less than $200 \mathrm{~ns}$. The spallation damage zones, $1-2 \mathrm{~mm}$ and almost at the central part of the samples without interaction with rarefaction-waves reflected along radial direction from these samples' lateral boundaries. In total two shots were performed at an impact speed of $200 \mathrm{~m} / \mathrm{s}$. Two samples with longer stress durations $(\mathrm{h}=0.585 \mathrm{~mm})$ were spalled. The others with shorter durations $(\mathrm{h}=0.45$ and $0.135 \mathrm{~mm}$ ) were cut off from their cross-sections and then fine-polished for close-up observations in order to check if microdamage occurred. 


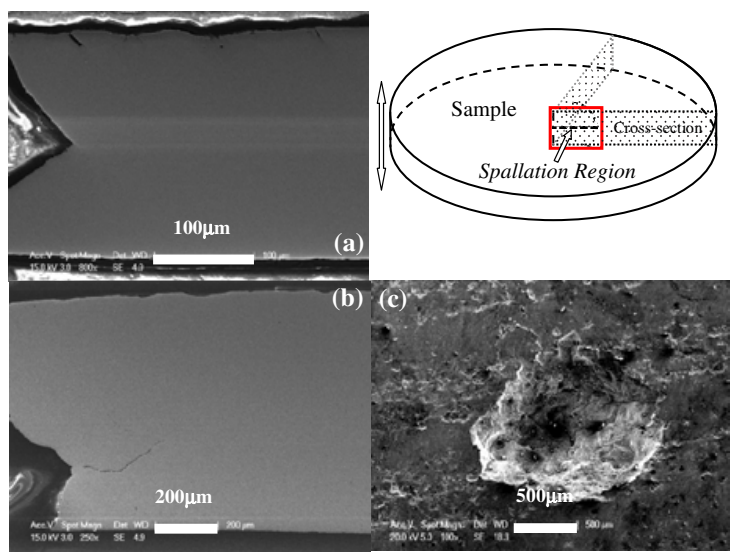

Fig. 2. Close-up observations of Zr-BMG samples impacted by flyers with various thickness at one shot $(V=200 \mathrm{~m} / \mathrm{s})$ : Part of cross-section of (a) the sample ( $h=0.135 \mathrm{~mm}$ ) and (b) the sample $(\mathrm{h}=0.335 \mathrm{~mm})$; (c) An over look of spallation occurred in the sample $(\mathrm{h}=0.585 \mathrm{~mm}$.).

\subsection{Experimental results}

Close-up observations with a high resolution scanning electronic microscopy (HRSEM) (FEI-Sirion NC HRSEM with $1.5 \mathrm{~nm}$ resolution) were performed on all the recovered BMG samples. For the spalled samples, fractographs of spallation surfaces were checked. For the samples with shorter durations, their cross-sections along these samples' radius direction were examined to make sure if microdamage occurred. As the samples are centre-symmetrical disks subjected to centre-symmetrical loading and the unloading effects of release waves initiate from the sample's lateral boundary, the resultant spallation as well as microdamage are likely to occur around the centre or the possible spallation regions. The close-up observations to all the tested samples illustrate that no damage, cracks or spallation was found in the regions beyond the possible spallation regions of each sample.

Figure 2 shows typical micro-observations of tested samples under a stress level of $3.18 \mathrm{GPa}$, in respect to various flyers' thickness. Figure $2 \mathrm{a}$ and $2 \mathrm{~b}$ are images of a half the possible spallation region each in cross-sections of the samples impacted by flyers of $0.133 \mathrm{~mm}(\mathrm{~h}=0.133 \mathrm{~mm})$ and $0.335 \mathrm{~mm}(\mathrm{~h}=0.335 \mathrm{~mm})$, respectively. An insert to the figure is a schematic of the location of micrograph shown in Fig. 2a and 2b. The dark shaded region in the cross-section (light shaded) indicates where spallation damage occurred. Figure $2 \mathrm{c}$ presents an overlook of the free surface of a recovered sample with a longer stress duration $(\mathrm{h}=0.585 \mathrm{~mm})$.

Figure 2a presents half of the possible spallation region in a sample impacted by a thin flyer (flyer's thickness, $\mathrm{h}=0.133 \mathrm{~mm}$ ). As shown in the figure, no microdamage can be observed in the possible damage region, even under higher magnifications. Figure $2 \mathrm{~b}$ is a typical sectional observation in a sample with a longer duration $(\mathrm{h}=$ $0.335 \mathrm{~mm}$ ). A main crack, about $200-250 \mu \mathrm{m}$ in length can be seen in the sample, from left (the sample's centre) to right (towards the lateral boundary of the sample) and along the wavy route between. However, only part of the crack is parallel to the sample surface. Whereas for a

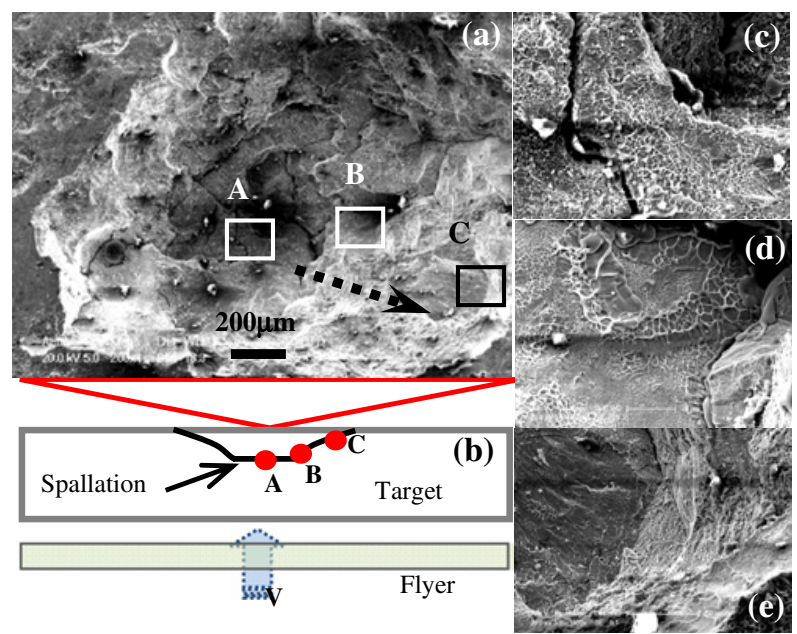

Fig. 3. A typical spallation occurred in the $\mathrm{Zr}$-BMG sample ( $\mathrm{V}=200 \mathrm{~m} / \mathrm{s}, \mathrm{h}=0.585 \mathrm{~mm}$ ): (a) An overlook of the spallation, squares $\mathrm{A}, \mathrm{B}$ and $\mathrm{C}$ denote different regions in the spallation surface, arrow's indicating is acclivitous direction of the surface; (b) A schematic of a cross-section of the spallation shown in (a); and enlarged imagines of (c) region-A; (d) region-B and (e) region-C.

sample impacted with thicker flyer $(\mathrm{h}=0.585 \mathrm{~mm})$, spallation seems to occur at the centre part of the sample, so that the spallation region looks like a pit with a size around $1-2 \mathrm{~mm}$ and the depth around 300-400 nm (Fig. 2c). Closeup observations shown in Figs. 2a, 2b and 2c illustrate that spallation occurred in the present Zr-based BMG samples is a damage evolution with stress duration increased under the same stress level

In order to understand further about microdamage of spallation occurred in the BMG samples, microscopic checking in detail was conducted on spallation surface of a spalled sample (tensile $=3.18 \mathrm{GPa}, \mathrm{h}=0.585 \mathrm{~mm}$ ).

Micrographs of this spalled sample $(\mathrm{h}=0.585 \mathrm{~mm})$ are shown in Fig. 3. Figure 3a presents a spallation surface remained in the sample after fragments had broken off. The spallation surface is pit-like, with a size of diameters 1$2 \mathrm{~mm}$ and depth $300-400 \mu \mathrm{m}$. In this figure the arrow is indicting an acclivitous direction of the spallation. Figure $3 \mathrm{~b}$ is a schematic of a cross-sectional image of the spallation surface. "A", "B" and "C" denote locations of the three squared regions in Fig. 3a. Enlarged images of these three regions are showed in Figs. 3c, 3d and 3e, respectively.

Figure $3 c$ is an enlarged image of region-A, which is at the bottom of the pit. According to knowledge of stress wave theory, region-A is located at the so-called spallation plane, on which the tensile stress amplitude is at the maximum value during loading. So the material in the spallation plane is under hydro-tensile stresses and subjects to a peak tensile stress along the sample's thickness. In this case the remained spallation surface in region-A exhibits typical vein-like patterns, which are in equiaxial shapes at microscales smearing over the region (Fig. 3c). However, in region-B whose location is on a higher level to region-A, besides some these vein-like patterns, several melt-down droplets and bolts can be seen in this region (Fig. 3d). This suggests that the fracture 


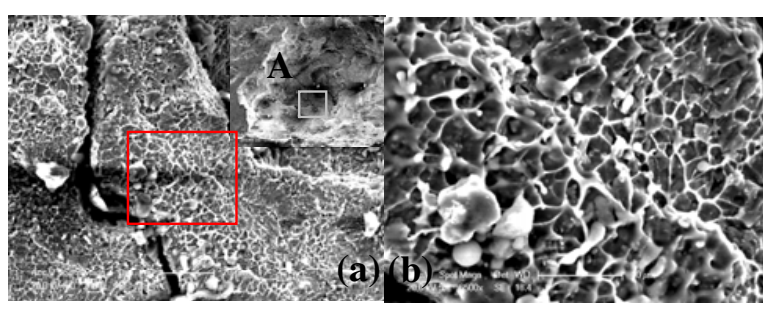

Fig. 4. (a) Fractography of the region-A as an insert showing; (b) Details of dimple structure with vein pattern in equal-axial shape in the squared region.

occurred at region-B may not be caused only by hydrotensile stresses like region-A. As for region-C, its location is far away from the bottom as shown in Fig. 3e, there is a relatively flat zone surrounded by rough slopes. Following these slopes a smooth place appears near to the free surface of the sample.

Actually, differences between fractographs shown in Figs. $3 c-3 e$ illustrate such fracture development from region-A to region-C. Fractographs of the three regions are examined in details as follows. Figure 4 displays the fractograph of region-A. An enlarged image of the squared region in the insert is shown in Fig. 4a, where veinlike patterns smear almost all over the region. Figure $4 \mathrm{~b}$ shows the details of the squared zone in Fig. 4a. The vein-like pattern is consisted with dimple cells of a nearly equiaxial shape at a scale of less than $10 \mu \mathrm{m}$, the cells' characteristic size. In particular, the dimple cell structure in this region seems to be built by micro-voids' stacking, so that under high magnifications this dimple structure is a porous structure with interconnected voids (Fig. 4b). Meanwhile, some small white particles, less than $100 \mathrm{~nm}$ in size, can be found out embedded in dimple cell walls. These dimple cells in equiaxial shape existed in regionA verifies that this region is under hydro-tensile stresses subjecting to a peak tensile stress along the sample's thickness. The porous micro-dimple cell structure in this region indicates that, under hydro-tensile stress in the spallation plane, the resultant microdamage should be microvoids, which would result in nucleation, growth and coalescence. As a consequence, spallation occurs.

Figure 5 displays micro observations to region-B. In this region, vein-like patterns are still in a nearly equiaxial shape and the cell size is about $100 \mu \mathrm{m}$ (Fig. 5a). Enlarged images of three small zones denoted by $\mathrm{a}, \mathrm{b}$ and $\mathrm{c}$ in the region are displayed in Figs. $5 b, 5 c$ and $5 d$, respectively. Figure $5 \mathrm{~b}$ shows details of zone-a. A melt layer and belts can be seen in the zone. Below the melt layer, there are vein-like patterns or dimple cell structures, with a cell size less than $3 \mu \mathrm{m}$. Figure $5 \mathrm{c}$ exhibits vein-like patterns in zone-b adjacent to the melt layer in zone-a. The veinlike patterns are in a size less than $5 \mu \mathrm{m}$ and are looked as if being formed in melt layers (Fig. 5c). Except for the ridges forming the veins, the fracture surface, on a close-up examination, is found to be highly smooth. Figure 5d shows small dimple cells in zone-c, where the dimple cell structure seems to be built in the melt layers. Actually, even though with equiaxial shapes, the vein-like patterns or dimple cell structures of region-B

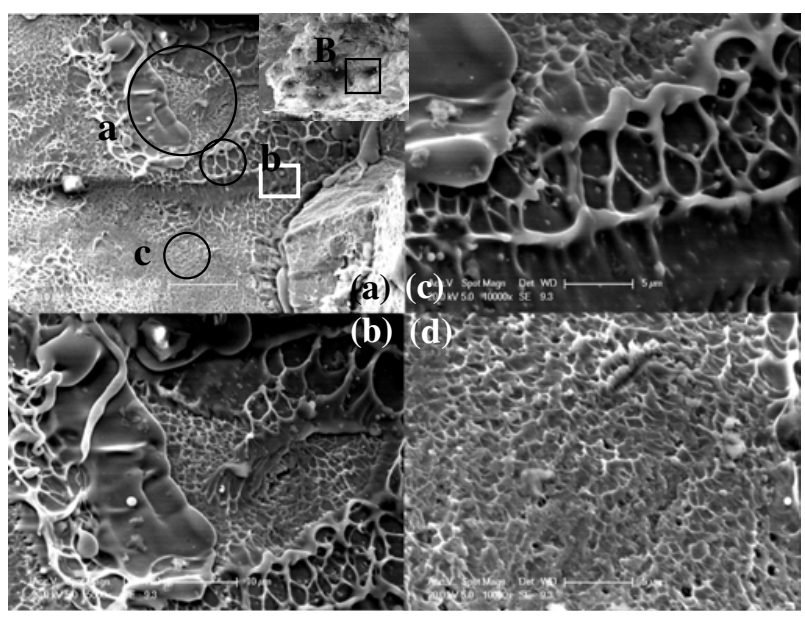

Fig. 5. Fractographs of the spallation in the sample $(\mathrm{V}=$ $200 \mathrm{~m} / \mathrm{s}, \mathrm{h}=0.585 \mathrm{~mm}$ ) as an inset showing: (a) Enlarged imagine of the region-B; (b) Enlarged imagine in zone-a, where vein patterns with melt droplets can be seen; (c) Details of zone-b following zone-a; (d) Dimples structures in zone c.

(Fig. 5) are no longer like those porous microstructures in region-A (Fig. 4). Similar phenomena to the melt layers and droplets, as main features in fracture surfaces, have been reported in previous studies on BMGs samples under tensile loadings $[13,22,23]$, where the fracture or samples' failing always occurred along a slip band or shear band at an angle with the tensile axis. This type of fracture is due to rapid softening of BMGs, which occurs close to the glass-liquid transition [13]. Particularly, Liu LF et al. [16] have also observed such melt droplets on fracture surfaces of BMG samples under plate shear loading. Fractographs with melt layers and vein-like patters in region-B clarify that fractures in the region do occur along directions with an angle to the tensile axis (along sample's thickness), or along a slip band that has an angle to the tension axis, since region-B deviates from the spallation plane. Moreover, it is suggested that the fracture surface of region-B is subjected to both normal (tensile) and shear stresses, rather than hydro-tensile stresses under the uniaxial strain state.

Figure 6 gives a set of micrographs in region-C, as shown in the insert. Figure 6a exhibits a close-up view of region- $\mathrm{C}$ and the arrow direction denotes the acclivitous direction from zone-a to zone-c. Whereas zone-a is relatively flat, zone-b is on the edge and zone-c close to the sample's surface. Figure $6 \mathrm{~b}$ shows zone-a in detail. The fractograph presents rough river-like structures smeared with vein-like patterns. Such a rough river-like structure is similar to those observed in previous studies [17], and should be induced by fast fracture that does not develop along with the direction normal to the impact direction. Figure $6 \mathrm{c}$ shows an enlarged view of zone-b, where voids of about $1 \sim 4 \mu \mathrm{m}$ in size seem to form a porous structure. However, the porous structure looks like honeycomb, quite different from that shown in figure $4 \mathrm{~b}$ with dimple structures. Such honeycomb structures have also been analyzed and regarded as a typical characteristic of transition from dimple to honeycomb structure as fast fracture or cracks 


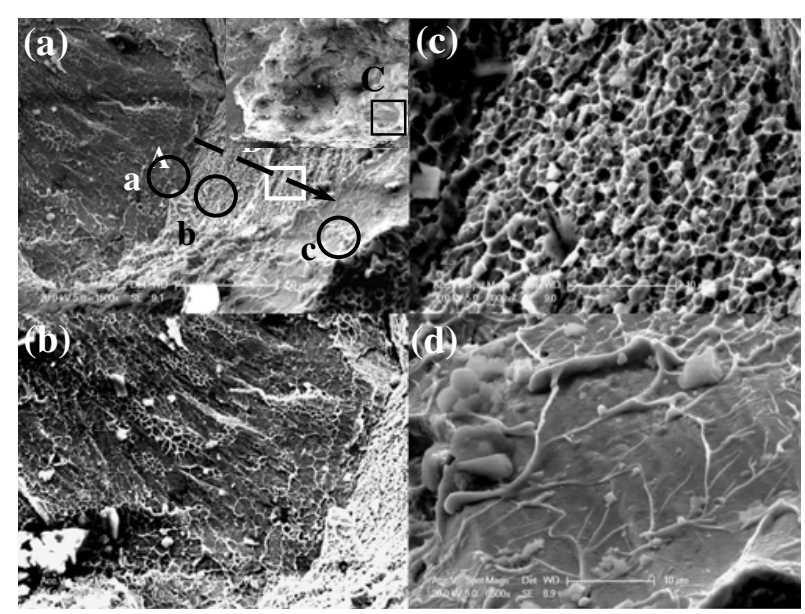

Fig. 6. Fractographs of the spallation sample $(V=200 \mathrm{~m} / \mathrm{s}$; $\mathrm{h}=0.585 \mathrm{~mm}$ ) as the inset: (a) Enlarged imagine of the region$\mathrm{C}$, near by the edge of the spallarion region. Along the direction of the arrow, zone-a local at the lower place, zone-b following zone-a and zone-c is very close to the sample's free surface; (b) Vein patterns in zone-a; (c) Dimples structures along the higher edge; and (d) Vein patterns and some melt droplets in zone-c.

developed in BMGs [17]. This transition is interpreted as, during fast fracture or cracks fast developing, both the size and the number of damage voids progressively increase, these voids form the honeycomb structures and the hackle patterns, thus promoting increased kinetic energy dissipation of the fast running crack and producing further more fracture surfaces. Therefore, the transition from dimple to honeycomb structures is ubiquitous in the region [17]. The observations in Figs. $6 \mathrm{~b}$ and $6 \mathrm{c}$ provide another evidence that, in this area, from zone-a to zoneb, cracks' fast running undergoes the transition. Figure 6d, however, exhibits a fractograph of zone-c, quite different from the other two zones shown in Figs. $6 \mathrm{~b}$ and $6 \mathrm{c}$. Typical vein-like patterns with melt droplets smear sparsely over the smooth surface within the whole area. Considering that melt droplets and ridges are results from either the adiabatic heating created by plastic flow [22] or free volume created by the existent hydrostatic tension [11]. This zone, with an angle less than $45^{\circ}$ to the sample's free surface, is very close to the boundary surface of the sample. Such fractograph is obviously produced due to stripping promptly from the sample surface.

Fractograph shown in Figs. 6b, 6c and 6d verifies again fracture occurred in region- $\mathrm{C}$ is a consequence of fast fracturing due to stripping of spallation occurred in region$\mathrm{A}$, or contributing of spallation occurred in region-A.

\section{Analysis and discussion}

Micrographs of region-A, B and C (Figs. 4, 5 and 6) in the fracture surface of the spallation sample had identical morphologies at different microscopic modes of fracture relating to different macroscopic behaviours, namely fracture stress, ductility, angle between the fracture surface and the tensile stress [13]. As illustrated in figure $3 \mathrm{~b}$ and figure 4, region-B and $\mathrm{C}$ deviated from the spallation plane (Fig. 3b), their micrographs, with the melt layer or droplets (Figs. 5 and 6), indicate that fractures in the two regions are both normal and shear stress dominated. Whereas, fracture occurred in region-A is hydro-tensile stress dominated, so spallation striations are visible over region-A. In particular, spallation striations in region-A can also be understood as the fracture occurred in the plane normal to tensile direction or the tensile axis. Such phenomenon shown in figure $3 \mathrm{~b}$ is consistent with the fracture model of BMG samples in the previous study of Inoue et al [23]. However, a slight difference between the case in Fig. $3 \mathrm{~b}$ and that indicated by Inoue et al [23] is that region-A in our current work is hydro-tensile stress (uniaxial-tensile strain) dominated rather than uniaxial-tensile stress controlled. Again, what presents in figure $3 \mathrm{~b}$ looks to be similar to that reported recently by Escobedo and Gupta [21], even though the spallation region in our work is within $10^{2} \mu \mathrm{m}$ in size, larger than the nano-scale zone suggested in the reference.

It is obvious that appearances of the topographical features on fracture surfaces depend on what conditions make them occur, being the final track of fracture process of nucleation, development and failure [13]. Fractograph of BMGs' samples under uniaxial tensile loading have been characterized in previous studies [13-15]. The veinlike pattern formed with ridges and flat, smooth surface in the BMG samples under uniaxial tensile loading were observed [11,22]. It is found that a main vein was nucleated around hard particle impurities by interphase cohesion or void formation [13]. More recently, very pretty vein-like patterns, a combined feature of veins and some radiating cores, appeared on the fracture surface of BMG samples under uniaxial tensile loading were reported [14]. Such vein-like pattern was suggested to be formed first by nucleated radiating cores due to tensile stress then by the beginning of crack/voids propagation under shear stresses.

Whereas in our current work the topographical features on spallation surfaces display vein-like patters formed with dimple cells of a nearly equiaxial shape. This vein-like pattern, as under hydro-tensile stress, is quite different from those patterns under combining normal and shear stresses. Clearly, the dimple structure without any tortuous displayed in region-A (Fig. 4) represents that spallation in the BMG samples is a typical dimpled rupture on the final track of spallation evolution. Some small white particles and small holes in the dimples' walls can be observed. Such dimple structures are formed under hydro-tensile stress loading. This morphology drops a hint that the dimples are nucleated from some hard particle impurities by interphase cohesion or void formation. As there is no shear stress to the sample during tensile stress loading, these small voids start to nucleate, grow, then coalesce, or to pull out under hydro-tensile stresses, leaving the dimples as seen in Fig. 4.

Generally, in region-A, the equiaxial vein-like patterns consisted with dimple structures exhibits a typical feature of fracture occurred under tensile stress in 1-D strain (Fig. 4). This feature illustrates that spallation occurred in current $\mathrm{Zr}-\mathrm{BMG}$ samples is typically dimple rupture. 
Furthermore this reveals that: (1) microdamage of the spallation occurred in the BMG samples is microvoids; (2) the spallation is resulted from microvoids' nucleation, growth and then coalescence; and (3) the time needed for microvoids nucleation is less than $100 \mathrm{~ns}$ with a stress amplitude of $3.18 \mathrm{GPa}$ (Table 2).

\section{Conclusions}

A Zr-BMG material subjected to dynamic tensile loading of the identical amplitude $(\sim 3.18 \mathrm{GPa})$ but different durations (83 $200 \mathrm{~ns}$ ) was studied for the purpose of revealing the micro-damage evolution process during spallation. With increasing stress duration, spallation microdamage in the BMG samples occurred at different levels. Spallation took place in the longest stress duration. For a spalled sample, fractographs of fracture surface in different regions, region- $\mathrm{A}$, region-B and region-C, were examined. Different fracture features in these three regions have illustrated that spallation occurred at region-A first, then fracture developed to region- $\mathrm{B}$, then finally turned to region- $\mathrm{C}$ due to stripping of spallation occurred in region- $\mathrm{A}$, or contribution of the spallation occurred in region-A. Among these regions, region-A was subjected to hydro-tensile stress, while deviating from the spallation plane, region$\mathrm{B}$ and $-\mathrm{C}$ were both normal and shear stress dominated. A typical equiaxial cellular pattern and a porous microstructure were found in the spallation surface within regionA. This indicates that (1) microdamage of the spallation occurred in the tested BMG samples is microvoids; (2) the spallation of the $\mathrm{Zr}-\mathrm{BMG}$ is resulted from nucleation, growth and coalescence of microvoids; and (3) the time needed for microvoids nucleation is less than $100 \mathrm{~ns}$ with a stress amplitude of $3.18 \mathrm{GPa}$.

\section{Acknowledgements}

Financial supports were from the NSFC (Grants Nos.: 10872206, 10721202, and 10725211), the National Natural Science Foundation of China-NSAF (Grant No: 10976100) and the National Basic Research Program of China (Grant No. 2009CB724401).

\section{References}

1. D.R. Curran, L. Seaman, and D.A. Shockey.,Physics Reports, 147, 5-6 (1987) 253-388.

2. M.A. Meyers, Dynamic behaviour of materials. 1994.

3. R. J. Clifton, International Journal of Solids and Structures,. 37, 1-2 (2000) 105-113.

4. Y.L. Bai et al., Journal of Applied Mechanics, 59, 3 (1992) 622-627.

5. H.L. Li, Doctoral dissertation, Peking University (1996).

6. S.D. Zhao et al., Proceedings of Shock Compression of Condensed Matters (1992) 523-526.

7. L.T. Shen et al., International Journal of Impact Engineering, 12, 1 (1992) 9-19.

8. Z. Ling and L.T. Shen, Journal De Physique IV, 134 (2006) 957-964.

9. A. Inoue et al., Materials Transactions Jim, 327 (1991) 609-616

10. D. Turnbull and M.H. Cohen, Journal of Chemical Physics, 34, 1 (1961) 120

11. F. Spaepen, Acta Metallurgica, 25, 4 (1977) 407-415.

12. A.S. Argon, Acta Metallurgica, 27, 1 (1979) 47-58.

13. C.A.Pampillo, Journal of Materials Science,10, 7 (1975) 1194-1227.

14. Z.F. Zhang, J. Eckert, and L. Schultz, Acta Materialia, 51, 4 (2003) 167-1179.

15. G. Wang et al., Intermetallics, 13, 6 (2005) 642-648.

16. L.F. Liu et al., Journal of Non-Crystalline Solids, 351, 40-42 (2005) 3259-3270.

17. M.Q. Jiang et al., Philosophical Magazine, 88, 3 (2008) 407-426.

18. S.M. Zhuang, J. Lu, and G. Ravichandran, Appl. Phys. Lett.,80, 24 (2002) 4522-4524.

19. S.J. Turneaure, S.K. Dwivedi, and Y.M. Gupta, J. Appl. Phys, 101 (2007) 043514.

20. F.P. Yuan, V. Prakash, and J.J. Lewandowski, J. Materials Research, 22, 2 (2007) 402-411.

21. J.P. Escobedo and Y.M. Gupta, J. Appl. Phys, 107 (2010) 043514

22. H.J. Leamy, H.S. Chen and T.T. Wang, Metall. Trans. 3, 3 (1972) 699

23. A. Inoue, Intermetallics, 8, 5-6 (2000) 455-468. 